\title{
UNLOCK: Uncovering and Noting Long-term Outcomes in COPD to enhance Knowledge
}

\author{
*Niels Chavannes ${ }^{a}$, Bjorn Stallberg ${ }^{b}$, Karin Lisspers ${ }^{b}$, Miguel Romanc, Ana Moranc, \\ Arnulf Langhammerd, Alan Crockette, Andrew Cave ${ }^{f}$, Sian Williams ${ }^{g}$, Rupert Jones ${ }^{\mathrm{h}}$, \\ Ioanna Tsiligianni', Thys Van Der Moleni, David Pricej
}

\footnotetext{
${ }^{a}$ Department of Public Health and Primary Care, Leiden University Medical Center, the Netherlands

c Spanish Primary Care Respiratory Group (GRAP), Spain.

${ }^{d}$ Department of Public Health and General Practice, Norwegian University of Science and Technology, Norway

e School of Population Health \& Clinical Practice, University of Adelaide, Australia

${ }^{f}$ University of Alberta, Canada

${ }^{g}$ International Primary Care Respiratory Group (IPCRG), United Kingdom

${ }^{\mathrm{h}}$ Peninsula Medical School, University of Plymouth, United Kingdom

'Department of General Practice, University Medical Center Groningen, the Netherlands

' Centre of Academic Primary Care, University of Aberdeen, United Kingdom
}

${ }^{b}$ Department of Public Health and Caring Sciences, Family Medicine and Clinical Epidemiology, Uppsala University, Sweden

Received 19th November 2010; accepted 22nd November 2010; online 29th November 2010

\author{
(c) 2010 Primary Care Respiratory Society UK. All rights reserved. \\ N Chavannes et al. Prim Care Resp J 2010; 19(4): 408 \\ doi:10.4104/pcrj.2010.00084
}

Keywords Chronic obstructive pulmonary disease, early diagnosis, prevalence, primary health care, screening, spirometry

\section{Background}

Primary care researchers co-ordinating databases from the United Kingdom, the Netherlands, Sweden, Norway, Spain and Australia are glad to announce the UNLOCK study: Uncovering and Noting Long-term Outcomes in COPD to enhance Knowledge. The group met at the IPCRG 2010 World Congress in Toronto and at a subsequent meeting at Stansted in September 2010.

\section{Objectives}

UNLOCK researchers recognise the need for establishing a common international dataset of relevant diagnostic and follow-up variables for COPD management in primary care. The primary purpose of the dataset is evaluation of the burden of disease (symptoms, limitations and exacerbations), the natural history of disease, treatment and follow-up, and co-morbidities in unselected primary care populations.

\section{Dataset}

The UNLOCK dataset should reflect the feasibility of data capture in real-world clinical practice, and ensure clinical relevance and completeness of the recorded data. Existing primary care datasets will be coupled and pooled to carry out analyses as appropriate to answer the group's research questions. Each country will create and maintain its own database which will be merged, as appropriate, in order to answer specific research questions.

\section{IPCRG Research Needs}

The UNLOCK researchers will tie-in with the recent IPCRG Research Needs Statement ${ }^{1}$ that identified a number of relevant issues in the diagnosis and management of COPD in different countries. Amongst others, pressing research questions currently considered are:

- Patient- and physician-related factors affecting outcomes and standards of care

- Impact and clinical meaning of constant diagnostic reclassification

- Comparison of different measures of health status and use of composite indices

- Relationship between health status and pulmonary function

- Long-term natural course of COPD in primary care

\section{Invitation to join}

Interested researchers are invited to collaborate with the group on a worldwide basis; a pre-requisite to collaboration must be the ability to contribute primary care patient data on COPD.

\section{Reference}

1. Pinnock H, Thomas M, Tsiligianni I, et al. The International Primary Care Respiratory Group (IPCRG) Research Needs Statement 2010. Prim Care Respir J 2010;19(Suppl 1):S1-20

* Corresponding author: Dr Niels H Chavannes, Department of Public Health and Primary Care, Leiden University Medical Center, Hippocratespad 21, Zone V0-P, PO Box 9600, 2300 RC Leiden, The Netherlands. Tel: +31 (0)71 5268444 Fax: +31 (0)71 5268259 E-mail: n.h.chavannes@lumc.nl 